\title{
Cerebral Venous Sinus Thrombosis: Two Case presentations with different clinical manifestations and multifactorial etiology
}

\author{
Dr Anusha Skandini Ganeshan ${ }^{1 *}$, Msc Dr Angel Alberto \\ Calderon Chongo ${ }^{2}$ \\ ${ }^{1}$ Senior Medical Officer Internal Medicine Department, \\ Seychelles Hospital, Victoria, Mahe, Seychelles \\ ${ }^{2} 1$ st degree: Specialist in Internal Medicine, Masters in \\ Infectious Diseases, Internal Medicine Department, Seychelles \\ Hospital, Victoria, Mahe, Seychelles.
}

\section{Citation: Dr Anusha Skandini Ganeshan; Msc Dr Angel Alberto Calderon Chongo2(2017) Cerebral Venous Sinus Thrombosis: Two Case presentations with different clinical manifestations and multifactorial etiology Open Science Journal 2(4).}

Received: $25^{\text {th }}$ July 2017

Accepted: $9^{\text {th }}$ September 2017

Published: $5^{\text {th }}$ October 2017

Copyright:@ 2016 This is an open access article under the terms of the Creative Commons Attribution License, which permits unrestricted use, distribution, and reproduction in any medium, provided the original author and source are credited.

Funding: The author(s) received no specific funding for this work.

Competing Interests: The author have declared that no competing interests exists.

\author{
*Corresponding Author: Anusha Skandini Ganeshan: \\ a_skandini2000@yahoo.com
}


Keywords: Cerebral Venous Sinus Thrombosis (CVST); Young females; Puerperium; Caesarean section under spinal anaesthesia; Oral contraceptives; Thrombophilia screening; Hyperhomocysteinaemia; Computer Tomography (CT); Magnetic Resonance Imaging (MRI); Magnetic Resonance Venography (MRV); Anti-Coagulant therapy; Low-molecular weight heparin (LMWH) and Warfarin

\section{Introduction}

Cerebral venous sinus thrombosis (CVST) is characterized by the formation of a clot in the intracranial dural venous sinuses or cortical veins, that drains blood from the brain and is less frequent than the thrombotic and embolic arterial infarctions $[1,2]$.

CVST is a rare condition affecting commonly young adults and children, primarily neonates $[3,4,5]$. In adults CVST is responsible for $3-4$ cases per million people per year [3] and $0.5-1 \%$ of all the strokes [2,3,7]. Whereas there is a higher incidence in neonates accounting for incidence of approximately 7 cases per million people per year according to studies in USA, Canada and Brazil $[3,4,8]$. Among the adult population, the female gender are predominantly affected than males with a ratio of $3: 1[3,5,9]$. Women tend to be at an increased risk particularly between the ages of $20-40$ years, mainly due to hypercoagulable state associated with the use of the oral contraceptive pills, pregnancy and the post-partum state[2,3,6,10]. Other common etiologies include hypercoagulable disorders, connective tissue disease, dehydration, infection, malignancy and trauma amongst others[11,12].

The variable and non-specific clinical presentation of CVST ranging from headache to focal neurologic deficits, altered sensorium and seizures, creates a diagnostic challenge for clinicians[2,3,7]. Many cases remain clinically undetected or misdiagnosed initially for other more common diseases, such as migraines, meningitis, sinusitis, ischemic or hemorrhagic stroke, thus consequently leading to complications and mortality $[3,5,7,10]$. Outcome in CVST with early diagnosis with the use of highly sophisticated imaging as Computer tomography (CT) scan of head and Magnetic Resonance Venography[6,12], and timely treatment with anticoagulation, carries better prognosis [2]. We discuss two clinical cases which are consistent and support the above statements from various researches and studies.

\section{Case 1 Report}

A 21 year old female presented to Accident and Emergency department with complaints of first episode of generalized tonic-clonic seizure, witnessed by partner. Patient was unaware of the event. She was well until the night before when she had experienced headache and transient numbness of left arm. 
Significant surgical history revealed that three weeks prior, she had undergone caesarean section under spinal anaesthesia, due to failure to progress in second stage of labour. She gave birth to a healthy child, without any post-natal complications. Noteworthy past medical history included Deep vein thrombosis (DVT) three years before.

General examination on presentation initially revealed fever of $\mathrm{T} 38^{\circ} \mathrm{C}$; Blood pressure of $110 / 60 \mathrm{mmHg}$; Tachycardia with heart rate of 110 beats per minute; other vital signs were within normal range. The significant clinical examination comprised patient being somnolent but arousable, oriented and cocorporative; GCS score of 14; Pupils were size 4 and sluggishly reactive bilaterally. Neurological examination revealed left arm monoparesis with muscle power of $2 / 5$ with decreased tone and reflex, all other limbs had normal power, tone and reflexes; Babinski negative; no neck rigidity or kerning's sign noted. On the abdomen she had a low-lying caesarean scar with a well - healed wound.

Initial working diagnosis: First episode of seizure for evaluation, with suspected Todd's paralysis and to rule out meningoencephalitis.

\section{Progress and Management:}

During the initial 2 hours of presentation, the patient developed further three episodes of generalized tonic-clonic seizure for about 1 minute duration, with frothing from the mouth, loss of consciousness with urine incontinence during the latter seizure, but no stool incontinence. The CT scan brain revealed normal findings. Following which the patient was re-assessed by neurologist on the ward, when she was noted to have developed left hemiparesis with left arm power of $0 / 5$ and left leg power of $1 / 5$ and facial asymmetry with inability to close left eye (7th cranial nerve palsy).

She was administered intravenous diazepam and intravenous phenytoin for seizure control. Hence with the working diagnosis most likely being meningoencephalitis, Intravenous methylprednisolone, IV Ceftriaxone, IV Acyclovir were commenced immediately and patient was transferred to Intensive Care Unit (ICU).

\section{Investigations:}

-Complete Blood count: WBC $12.5 \times 10^{3}$ uL (high); Granulocytes $81.3 \%$ (high); Hgb 12.7 g/dl; Platelet 320x103 uL; MCV 83.1 fL; PT/INR 1.27s; aPTT $25.7 \mathrm{~s}$

-Erythrocyte sedimentation rate (ESR) $17 \mathrm{mmHg}$; Lactose dehydrogenase (LDH) 386u/l; C-Reactive Protein (CRP): $54.6 \mathrm{mg} / \mathrm{l}$ (high)

-Electrolytes, Renal \& Liver parameters: : within normal limits (Cr 57).

-HIV, Hepatitis A,B,C Screening- Negative

•Urine culture: White Blood Cell nil; Red Blood Cell nil, no growth; Blood culture-no growth

-Chest X-ray, ECG and Echocardiogram - normal; US Abdomen-normal.

-Lumbar Puncture Cerebrospinal fluid (CSF) results:

- Clear; White Blood cell: $15 / \mathrm{mm}^{3}$ (neutrophils); Red blood cells $63 / \mathrm{mm}^{3}$

- Protein $14.3 \mathrm{mg} / 100 \mathrm{ml}$ (normal); Glucose $4.03 \mathrm{mmol} / \mathrm{l}$ (normal)

- Cytology: scanty cellularity with occasional lymphocytes. 
-Magnetic Resonance Imaging (MRI) with Angiogram of Brain: done on following day:

oMultiple right frontoparietal focuses of brain edema with ischemic lesion, suggesting partial right mid-cerebral artery (MCA) infarct

oRight transverse sinus partially occluding thrombus cannot be excluded.

From CSF results, meningoencephalitis was ruled out by neurologist. Following MRI finding, right MCA territory infarct was the second primary diagnosis, however following a joint consultation with neurologist and radiologist, final diagnosis concluded was highly likely Cerebral Venous Sinus Thrombosis. Further workup was done to rule out autoimmune diseases / embolic event / thrombophilia.

\section{Diagnosis:}

Cerebral Venous Sinus Thrombosis - Transverse sigmoid sinus thrombosis.

Further Investigations: Thrombophilia / autoimmune disorder screening:

Homocysteine - $38.9 \mathrm{umol} / \mathrm{l}$-High (normal range 0.0-14.4)

Protein S - 39\% - Low (normal range 60-123)

Anti-thrombin III - $123 \%$ - High (normal range 83-118)

Protein $\mathrm{C}$ and Fibrinogen- normal; Serum Beta-2 Microglobulin- normal

Anti-cardiolipin IgG, IgA, IgM - within normal range; Anti-nuclear antibodies (ANA), Double-strand DNA- negative.

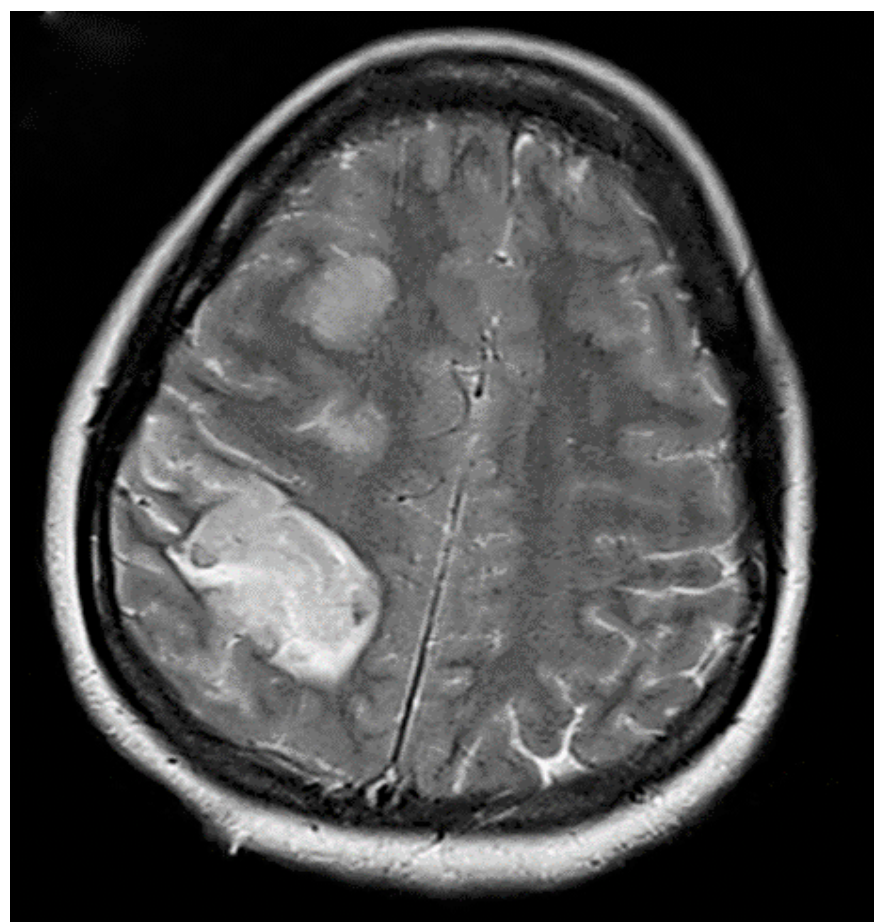

Figure 1: MRI axial view (T2-weighted-Fluid-Attenuated Inversion Recovery T2-FLAIR) illustrates multiple right frontoparietal white matter and cortical hyperintensities with focuses of brain edema with ischemic lesion 


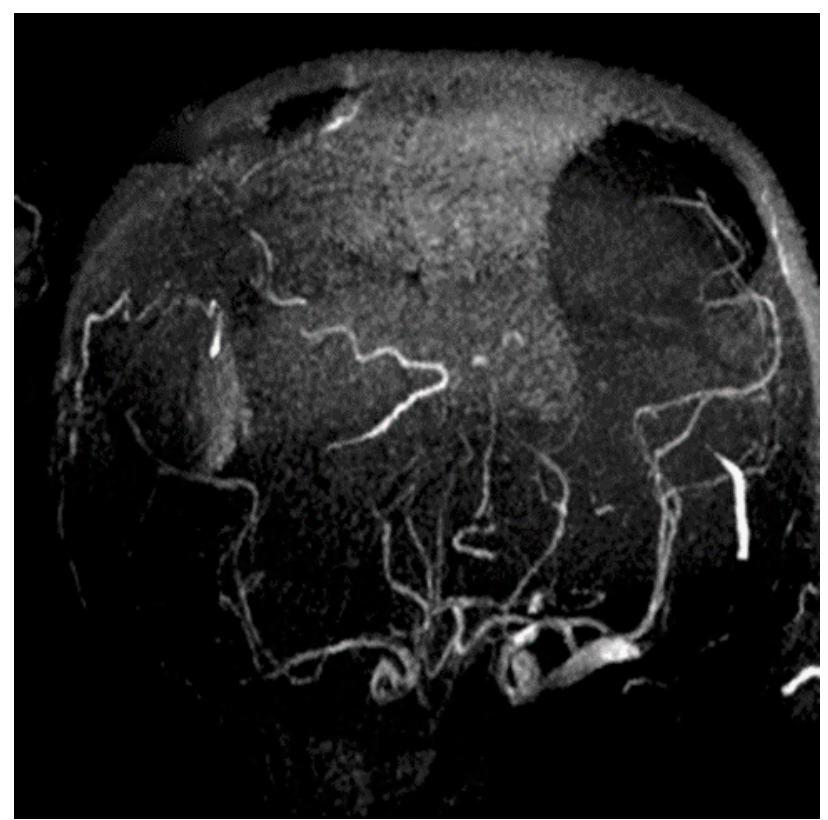

Figure 2: demonstrates that right transverse and sigmoid sinus are asymmetrically narrowed, but flow is present (arrow).

\section{Progress:}

First day in ICU, the patient developed recurrent multiple right-sided focal seizures and status epilepticus, she was hence sedated and placed on ventilator support and started on IV phenobarbitone. In view of MRI findings, the dose of methylprednisolone was increased, and commenced on IV mannitol (for brain edema) and subcutaneous Low-Molecular Weight Heparin (LMWH) Fraxiparin $0.6 \mathrm{ml}$ BD. On day 6, she was stable and hence transferred to Internal Medicine Department, she was noted to have persistent headache and left hemiplegia on neurological examination. She progressed well on the ward, gradually regaining power in left-sided limbs to $3 / 5$, with support of physio and occupational therapy. Medications were continued, with tapering down of mannitol over 10 days, ceftriaxone continued for completion of 10 days, in view of suspicion of underlying infection as one of the possible contributing factor of the event (though source was not found); Analgesics added for headache. Serum Phenytoin and Phenobarbitone levels were checked regularly and dose of medication adjusted accordingly.

\section{Further Imaging:}

1)Follow-up CT Brain (day 3): haemorrhagic infarction in right cerebral hemisphere, with increased peri-focal edema, and increased mass-effect with right-to-left midline shift of about $5 \mathrm{~mm}$. 


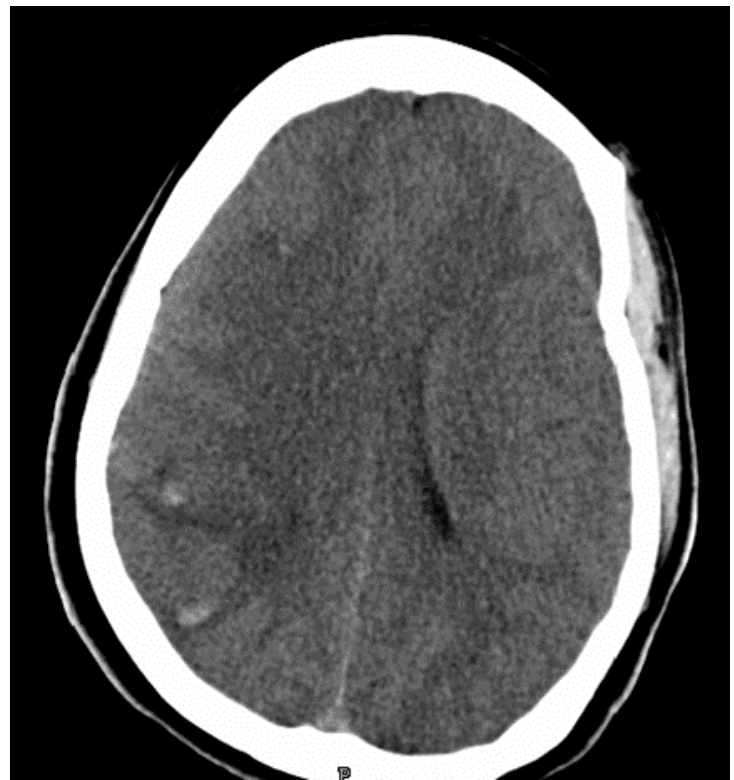

Figure 3: Axial view of plain CT Brain image: showing ischemic infraction in the right cerebral hemisphere with multiple hyperdensities suggestive of acute haemorrhage within the infarct area (arrow); There is greater mass effect as compared to MRI on previous day, resulting in greater compression of the right lateral ventricle with right-to-left midline shift of about $5 \mathrm{~mm}$.

2) Repeat MRI Brain (day 10): Absent flow in right transverse sigmoid sinuses suggestive of Right Transverse sigmoid sinus thrombosis with right cortical frontoparietal ischemic changes with petechial haemorrhagic components and diffusion restriction associated with prominent perifocal edema, mild mass-effect and lateral ventricle compression.
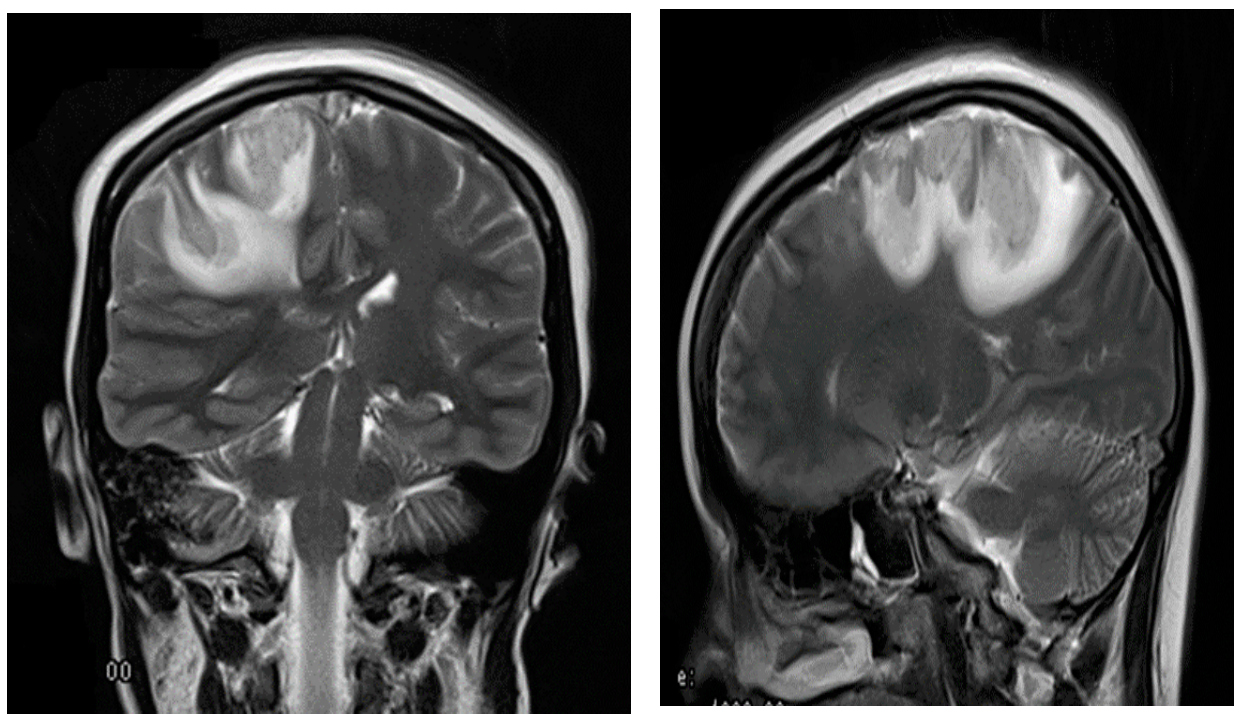

Figure 4 and Figure 5: Represents coronal and sagittal view images, respectively, of MRI T2-weighted scan: both demonstrates right cortical frontoparietal ischemic changes with petechial haemorrhagic components and diffusion restriction associated with prominent perifocal oedema with mass-effect and lateral ventricle compression. 

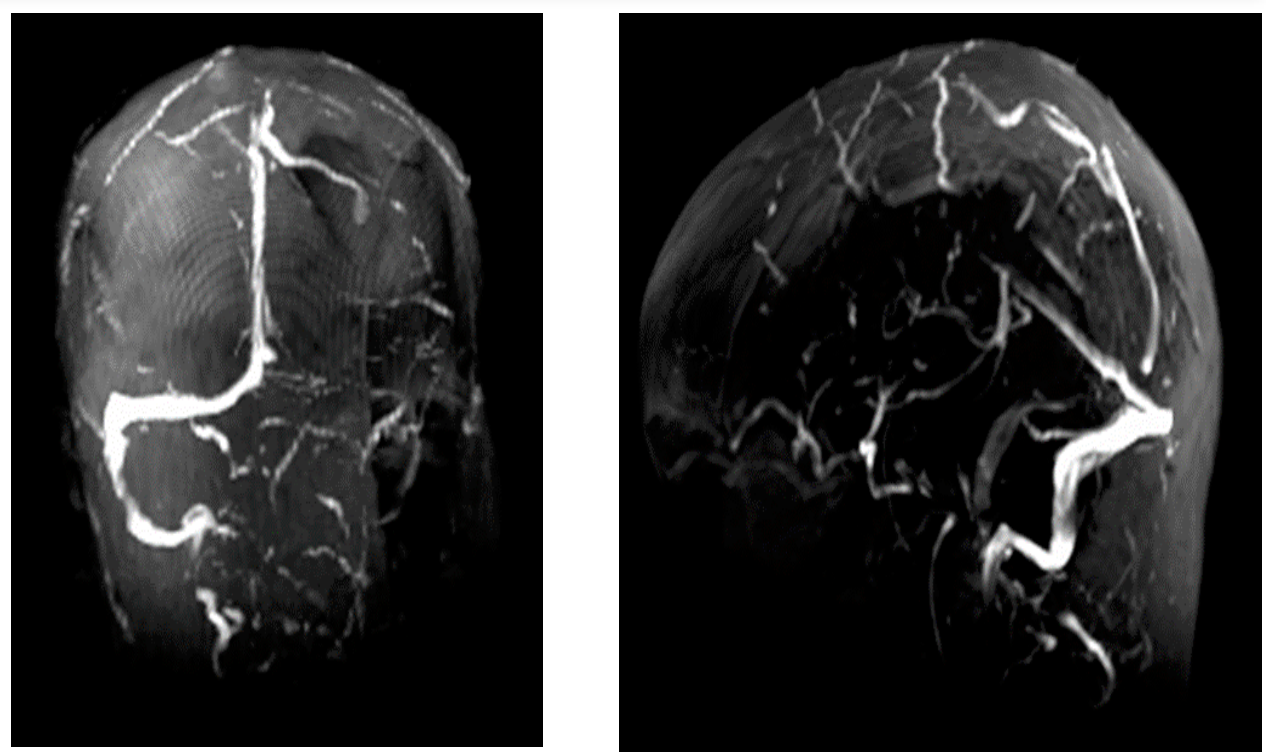

Figure 6 and Figure 7: Shows Magnetic Resonance (MR) Venography images demonstrating absence of flow in the right transverse sigmoid sinuses suggestive of Right Transverse Sigmoid Sinus thrombosis.

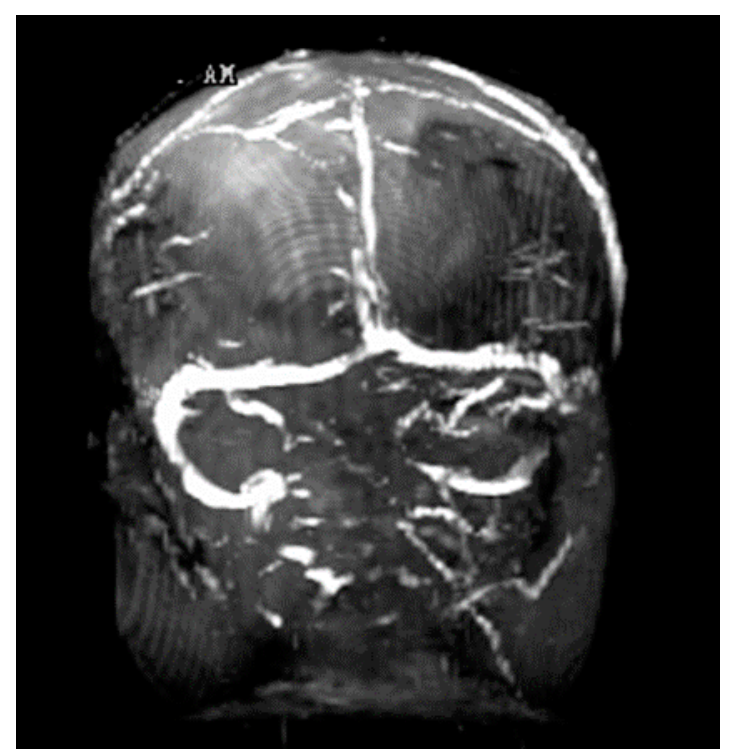

Figure 8: MRI Venography shows restored flow through the Right Transverse sigmoid sinus following 3 months of anti-coagulation treatment

\section{Further management and Follow-up:}

On day 12 , due to recurrence of right-sided focal seizure, Levetiracetam was started by neurologist, and phenobarbitone was tapered down and discontinued. On day 14, she was started on oral anti-coagulation therapy - Warfarin, with regular $\mathrm{PT} / \mathrm{INR}$ monitoring and dose adjusted accordingly. Upon making remarkable recovery, she was discharged on day 20, on Warfarin, Phenytoin and Levetiracetam.

She is still on anticoagulation with regular follow-up to date by physician and neurologist, with PT/INR check. She will continue anti-coagulation for life, due to multifactorial risk factors leading to her CVST. She has fully recovered 
with only minimal residual weakness on left arm remaining. She had a repeat MRI Angiogram after 3 months, which revealed complete restoration of normal blood flow in the cerebral venous sinuses.

\section{Case 2 Report}

A 25 year old female presented to Accident and Emergency Department with complaints of sudden onset of inability to speak, facial asymmetry with loss of muscle power on the right arm and occasional muscle twitching on right arm, which she noticed as she woke up that morning. She was previously in good health with only significant past medical history of migraine, which was well controlled with simple analgesics. Surgical history comprises caesarean section three years ago. Substantial medication history included the patient being on oral contraceptive pills (microgynon) for the past two years.

General examination revealed Blood pressure of $157 / 88 \mathrm{mmHg}$; Heart rate 92 beats per minute; other vital signs were within normal range. The clinical examination that was significant to note was on general appearance patient looked anxious, but she was conscious and oriented, obeyed command. She was aphasic but responded to question with hand signs and head nod gestures. Neurological examination revealed facial asymmetry on right-side of face with loss of forehead creases on right side with deviation of the mouth and tongue to the left side, indicative of facial nerve paralysis on the right side. Right arm muscle power was $0 / 5$, hypotonia with diminished reflex. Other limbs were of normal power and tone. Babinski was positive on the right leg.

\section{Investigations:}

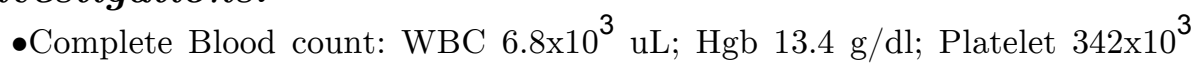
uL; MCV 83.1 fL; PT/INR 1.53s;

-Erythrocyte Sedimentation Rate (ESR) 2mmHg; C-Reactive Protein (CRP) $4.5 \mathrm{mg} / 1$

-Electrolytes, Renal \& Liver parameters: : within normal limits (Cr 60).

-D-dimer, fibrin degradation products (FDP) - Negative

-HIV, Hepatitis A,B,C Screening- Negative

-Urine micro: White Blood Cell nil; Red Blood Cell nil; urinalysis was normal.

-Chest X-ray - Normal

-ECG and Echocardiogram - normal.

-CT Brain on admission: Normal study

-Magnetic resonance imaging (MRI) of Brain was done on following day of admission: revealed high T2 signal in the left parenchymal gyrus, suggestive of ischemic infarction. No evidenced of raised intracranial pressure.

- Magnetic Resonant Venography on day 2: narrowing of the Superior sagittal sinus, suggestive of partial occlusion by thrombus.

\section{Diagnosis:}

Cerebral Venous Sinus Thrombosis- Superior Sagittal Sinus thrombosis. 


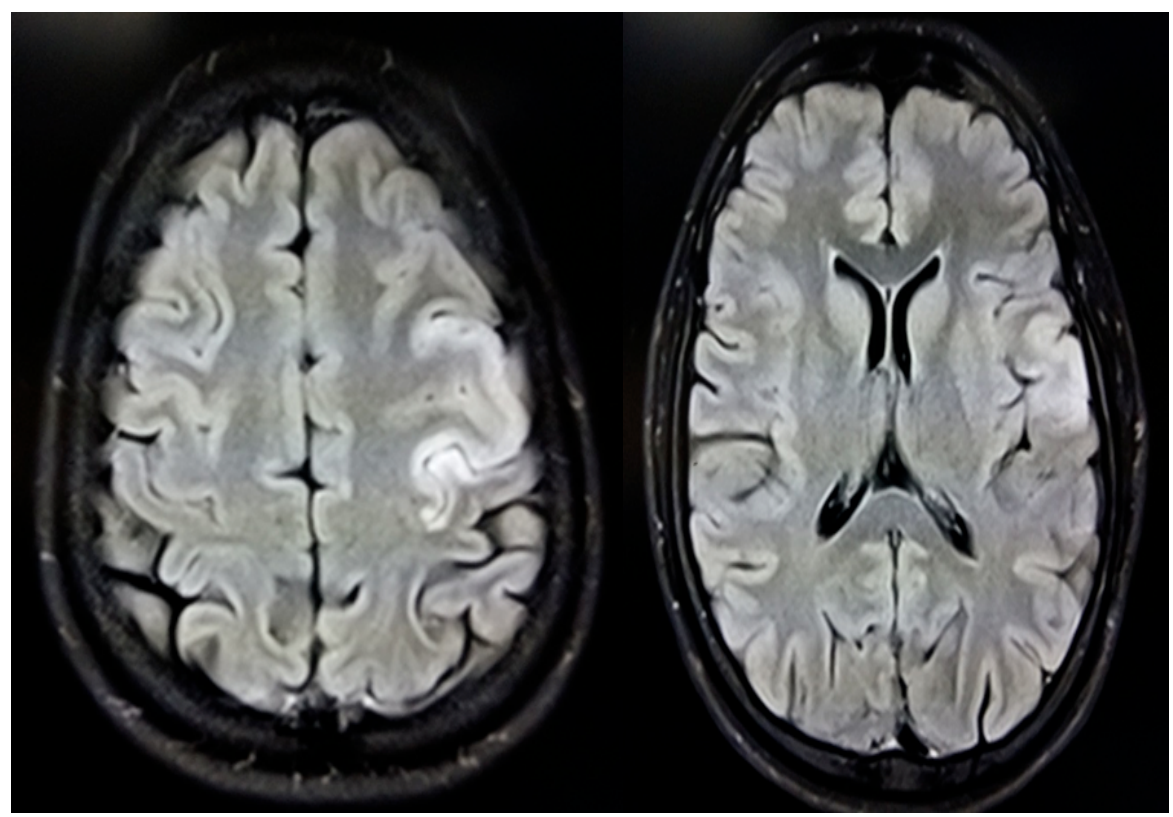

Figure 9 and Figure 10: illustrates MRI T2-weight axial view images demonstrating hyperintense (high signal) in the left frontal lobe, suggestive of ischemic infarction.
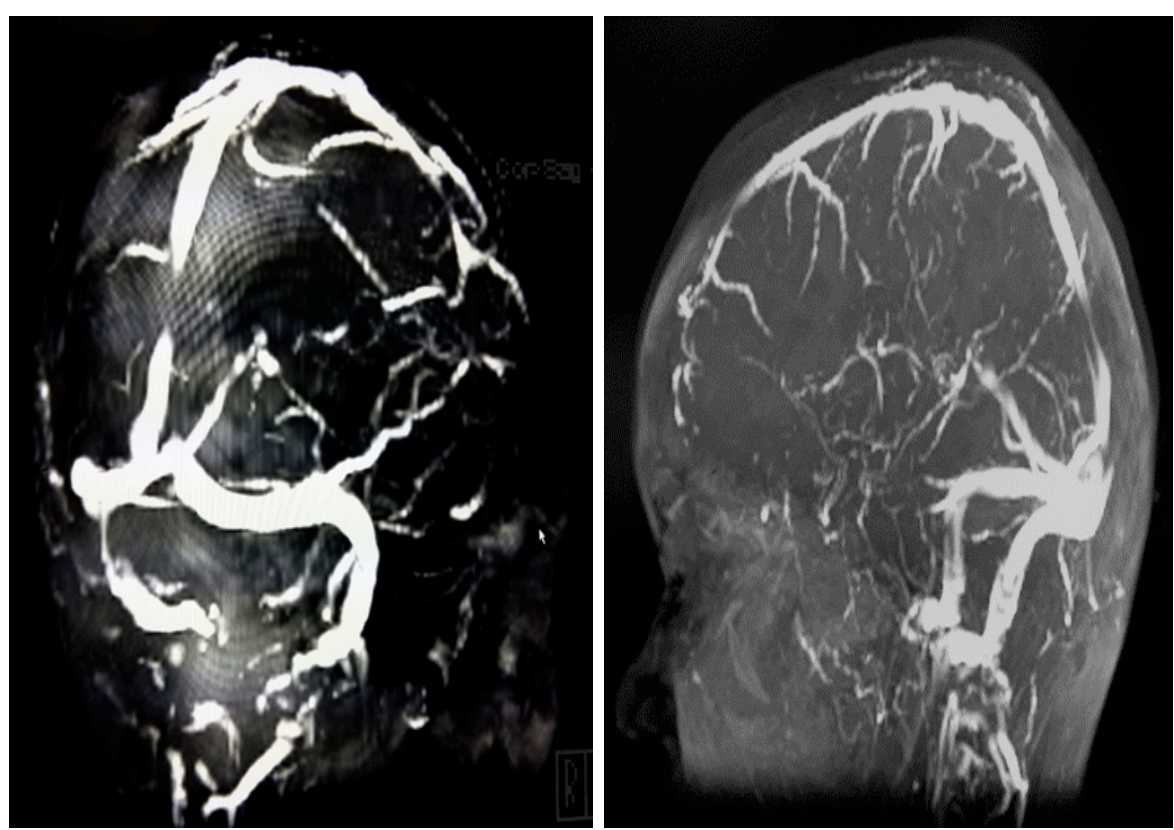

Figure 11 and 12: illustrates Magnetic resonant Venography clearly demonstrating narrowing of the Superior sagittal sinus, suggestive of partial occlusion by thrombus.

Further Investigations: Thrombophilia / autoimmune disorder screening:

•Homocysteine - $46.6 \mathrm{umol} / \mathrm{l}$ (normal range 0.0-14.4) - (High)

-Protein S, Anti-thrombin III, Protein C and Fibrinogen, Anticardiolipin- All within Normal range; ANA, Ds-DNA-negative.

- Repeat MR Venography on day 7: high signal flow in the left frontal lobe is less evident as compared to previous MRI indicative of subacute 
phase of evolution of ischemic infarction. Persistent partial narrowing of the superior sagittal sinus flow.

\section{Management:}

Patient was admitted to Internal Medicine Department with an initial working diagnosis of possible ischemic stroke and commenced on aspirin, oral contraceptive pills were ceased; physiotherapy, speech and occupational therapy were initiated. Following MRV results, CVST was made as the primary diagnosis, and with positive risk factor of oral contraceptive use, she was initiated on therapeutic dose of Low-molecular weight heparin (Fraxiparin) followed by warfarin. On day 3 patient developed severe headache on a scale of 8/10, hence analgesics were added.

Patient recovered remarkably well with significant improvement of neurological deficit. She was discharged on day 11 on warfarin and to continue physio and speech therapy. Upon discharge, she had complete resolution of facial palsy, but residual deficit in right arm with muscle power of $4 / 5$ and mild dysphasia remained.

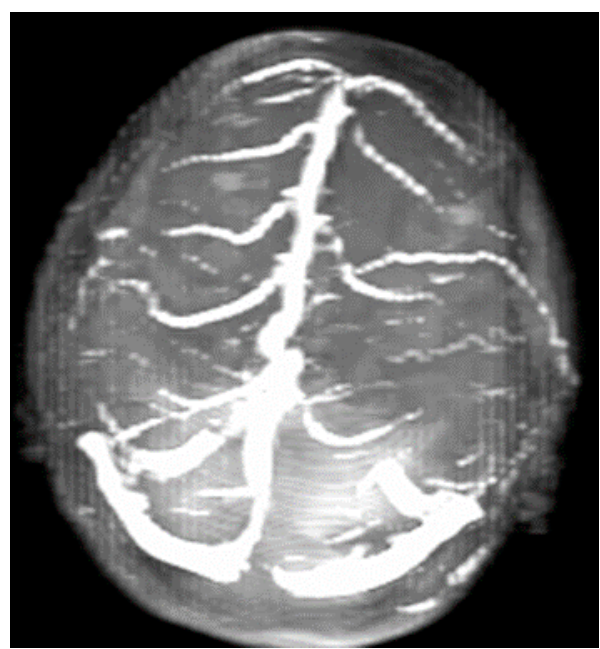

Figure 13: illustrates Magnetic resonant Venography with complete resolution of superior sagittal sinus thrombosis and restoration of normal flow.

\section{Follow-up:}

Patient was followed-up in outpatient clinic by neurologist and physician with regular PT/INR checks and warfarin dose adjusted accordingly. The patient made complete recovery with no residual neurological deficit, however she continues to have intermittent headaches, controlled by oral analgesics. A control MRI brain with MR Venography was done after 4 months, which revealed complete resolution of superior sagittal sinus thrombosis with restoration of normal flow, as illustrated on figure 13. After 7 months, warfarin stopped by neurologist and changed to aspirin and she is followed-up regularly to this day. 


\section{Discussion}

Cerebral Venous Sinus Thrombosis (CVST) is a rare and often underdiagnosed condition affecting commonly young adults and children[3,4,5]. Young female are more at risk than males, with ratio of 3:1[9] due to pregnancy, spinal anesthesia administration for caesarean section or during labour, postpartum state and hormonal therapy (oral contraceptives)[6,9]. The reported incidence of CVST during pregnancy and puerperium in women in developed countries is 11.6 per 100,000 deliveries per year, accounting for $6-64 \%$ of pregnancy-related strokes and the incidence is believed to be much higher in developing countries [7]. Common cause of CVST includes prothrombotic condition secondary to hereditary thrombophilia such as factor $\mathrm{V}$ Leiden mutation, prothrombin gene G20210A mutation, absence of anti-thrombin III, and anti- $\beta 2$ glycoprotein-I antibodies, absence of protein $\mathrm{C}$ and/or protein $\mathrm{S}$ and hyperhomocysteinaemia $[3,6]$; Other risk factors include lumbar puncture, intracranial infection, dehydration, sepsis and systemic infection, surgery[9], connective tissue disorders $[6,12,13]$ and malignancy[10].

In both cases discussed above, it is evident that both patients had multifactorial, three or more, etiologies contributing to CVST. Note that both patients were young females in their $20 \mathrm{~s}$ and both had hyperhomocysteinaemia as common risk factors. Additional risk factors in the first patient included her puerperal state, recent caesarean section under spinal anesthesia, suspected infection (confirmed by fever, raised WBC, Granulocyte count and CRP), low levels of Protein S and a past history of DVT. In the second patient with partial thrombus, the additional risk factor elicited was her use of oral contraceptive pills.

The most commonly affected venous sinuses are superior sagittal sinus, followed by the transvers sinus with the frequencies of $62 \%$, and $41 \%$, respectively[9,10]. There is an ongoing study in Seychelles to determine the cause of hypercoagulable state leading to high prevalence of Deep Vein Thrombosis (DVT) and Pulmonary Embolism (PE) among the Seychelles population. Stoke (arterial infarction) is very common condition in clinical practice among the Seychelles population primarily due to co-morbid risk factors as hypertension, diabetes mellitus and dyslipidaemia. Over the past 10 years, there has been two cases of cavernous sinus thrombosis in males, however there are no reported cases of main CVST in adults except for these two cases, either due to underdiagnosed cases or the rare occurrence of the disease.

\section{Clinical Features:}

Cerebral Venous Sinus Thrombosis is difficult to diagnose due to the variety in clinical symptoms[6].

$>$ It is estimated that up to $80-90 \%$ of CVST patients first present with either focal, diffuse or migraine-type headaches[3].

$>$ Depending on the location of thrombosis, there are variable clinical manifestations including: focal neurological signs such as hemiparesis, diplopia, or visual impairment, aphasia, altered level of consciousness, focal or generalized seizures $[6,7,9]$ that indicate involvement of brain parenchyma[5]. 
Papilledema may also develop[3,6].

> The less frequent presentation includes rapidly progressive illness with deepening coma, and pyramidal signs, due to extensive involvement of the deep cerebral veins[14].

The presence of variable and non-specific symptoms often times may be confused for other more common diseases, such as migraines, meningitis, sinusitis, ischemic or hemorrhagic stroke[3,7]. In pregnant women, such clinical presentations can be easily confused with the normal pregnancy state or related complications as eclampsia, making it difficult to diagnose and treat this disease in a timely manner, thus potentially life-threatening for the patient and fetus[7].

This supports the variable clinical presentations of the two patients discussed in this article, with the first case presenting with fever, headache, recurrent seizures, followed by left hemiparesis and inability to close left eye, proceeded by status epilepticus. The second case presented with acute onset of right monoplegia, right facial nerve palsy, aphasia and later proceeded by headache. Hence in both cases, primary diagnosis was of other more common diseases, as meningoencephalitis, seizure with Todd's palsy, and ischemic stroke, respectively.

\section{Pathophysiology CVST:}

Cerebral veins are thin, valveless structures that do not contain smooth muscle. Venous infarcts occur due to cerebral vein occlusion by thrombus or external compression. Subsequently, first the venous pressure continues to rise leading to cortical edema and the appearance of vasogenic edema on im $\neg$ aging[15], as illustrated in the initial MRI images in both cases above. The CSF is normally absorbed by the arachnoid villi and drains into the superior sagittal sinus, however due to occlusion of the cerebral venous sinuses, subsequent development of raised intracranial pressure occurs, this constitutes the second mechanism[10]. If the rate of rise in venous pressure is rapid, the friable valveless ve $\neg$ nous vasculature is unable to withstand the pressure, ultimately leading to paren $\neg$ chymal haemorrhage[15], as in case one above.

\section{Investigations:}

A clinical suspicion of CSVT is the first step of diagnosis[9], however further investigations must be done to confirm the diagnosis and to establish any predisposing etiologies[10,13].

1. Conventional Computed Tomography $(\mathrm{CT})$ : often remain the first imaging modality to be used, simply due to availability and also to exclude other conditions such as intracerebral hemorrhage/ abscess/[13] space occupying lesion. CT scanning with contrast enhancement may demonstrate an 'empty delta sign', is a useful radiological sign in superior sagittal sinus thrombosis [10], however it is present in only $10-20 \%$ of cases. CT is entirely normal in $10-20 \%$ of cases with proven CVST[13], as is the case in both patients discussed above.

2. Lumbar puncture: which maybe abnormal in up to $84 \%$ of cases (include raised CSF pressure, increased protein content, the presence of red blood cells 
and pleocytosis[13]), and may also be helpful in ruling out other diagnoses [10], as done for the first patient.

3. MRI Brain combined with MR Angiography (MR Venography-MRV): is by far the most sensitive and specific method for the diagnosis of CVST and is considered as gold standard [8,13]. On MRI T1 hyperintense thrombus or loss of T2 flow void effect may be found within the dural sinus. MRV can show the consequences of thrombosis such as cerebral edema, infarction and hemorrhage as well as the anatomy of the disturbed venous circulation,[13] as illustrated in MRV images for both patients above.

4. Other investigations: are directed towards demonstrating the underlying cause. Clinically obvious cases such as local infection or head injury may be selfevident, whereas extensive investigations are needed in the 'idiopathic' cases. Suspicion of malignancies or connective tissue diseases should be confirmed with appropriate tests such as chest X-ray or other imaging, inflammatory markers, autoantibodies or tissue biopsies[13,17].

5. Coagulation studies: are important and the investigations should include all hereditary thrombophilia screening stated above in etiology [13,17]. All these investigations should be done before starting anticoagulation[13], same done in both cases discussed.

\section{Management:}

Immediate management of CSVT includes strategies aiming to recanalize the thrombosis, and to prevent further complications with the treatment of underlying causes.

Anticoagulation: with heparin (LMWH) forms the mainstay of treatment, despite the risk of hemorrhage into venous infarcts, or even in the presence of an existing hemorrhagic venous infarct $[6,9,10]$, as demonstrated in management of both cases above. Anticoagulant therapy acts by reduction in thrombus propagation and aided in early recanalization of the thrombus[4].

$>$ Oral anti-coagulant, Warfarin, is usually continued for 6 months, except when there is a known prothrombotic condition in which treatment may have to be life-long[13], with a target INR of $2-3$ in order to reduce the risk of recurrent cerebral and extracranial thromboses $[9,10,17]$. Same management established in both cases above.

$>$ Endovascular approaches: as urokinase and tissue plasminogen activator may be used as thrombolytic treatment if the patient's condition worsens with progressive neurologic deterioration despite sufficient heparinization[6,9].

$>$ Mechanical thrombectomy: may also be considered in some patient with neurologic deterioration[6,9], though this is subject to availability of facility.

$>$ Special interventions to reduce significantly raised intracranial pressure: include acetazolamide, steroids, repeated therapeutic lumbar punctures, mannitol, shunt procedures, surgery and barbiturate induced coma[9,13]. 
Other symptomatic treatments: such as antibiotics, anticonvulsants, anti-emetics and analgesia will depend on the circumstances[6,13], as in case one above.

\section{Complications:}

$>$ Seizures

$>$ Venous infarct and Intracranial Hemorrhage[6,7] associated with varying neurological deficit

> Intracranial hypertension presenting with headache, visual loss, and/or deterioration in level of consciousness[9]

$>$ Venous Thromboembolism (DVT or PE)

$>$ Risk of recurrence CVST[3,18]

$>$ Mortality[18]

\section{Mortality/Morbidity:}

The prognosis of CVST was once very poor, with a mortality rate of up to $30-50 \%$, however most studies now show good outcomes. With newer treatment modalities, it is estimated that there is only a $5-10 \%$ mortality rate as a result of CVST $[3,6]$. Studies indicate that there are predictors of increased mortality, namely: depressed consciousness, altered mental status, papilledema, seizures, underlying malignancy, sepsis, advanced age, thrombosis of the deep venous system, right hemisphere hemorrhage, or posterior fossa lesions $[3,6,7,10,13]$.

\section{Conclusion}

CVST is thus far an uncommon condition. CVST can be easily misdiagnosed due to its diverse, non-specific symptoms. As in our discussion above, in the first case, the patient presented with fever, generalized tonic-clonic seizures, which progressed to status epilepticus, and left hemiparesis. The first CT brain was normal and basic blood investigations revealed raised WBC, granulocyte count and CRP, hence all these mislead the clinician in making the initial diagnosis as being meningoencephalitis associated with seizures and Todd's paralysis. Likewise in the second case, patient presented with acute onset of aphasia, and left monoparesis and right facial weakness, again first CT brain was normal, which lead the clinician to suspect stroke as the primary working diagnosis, given the commonality of the latter in Seychelles.

However, in both cases, prompt diagnosed of CVST (Transverse sigmoid sinus thrombosis and Superior sagittal sinus thrombosis, respectively) was made, on confirmation by gold standard neuroimaging (MRI brain and MRV) within 24-48 hours of presentation, respectively. Upon confirmation of CVST, full screening was done to rule out underlying predisposing etiologies including thrombophilia/ autoimmune diseases/ embolic event. Both patients had more than three risk factors, with the first patient being high-risk. Common risk factors included, both being females, young in their 20s, and on thrombophilia screening, both had hyperhomocysteinaemia. Additional risk factors in the first patient included her puerperal state, recent caesarean section under spinal anaesthesia, underlying infection, low levels of Protein S and a past history of 
DVT. In the second patient other risk factor was her use of oral contraceptive pills.

Nonetheless, as established in numerous studies and evident in both patients, despite multiple significant complications in the first patient, the early diagnosis and subsequent prompt initiation of treatment with anti-coagulation and adequate supportive treatment, yields good prognosis. Thus it is imperial for clinicians to keep CVST as a differential diagnosis especially when treating young females patients, in reproductive age on oral contraceptives, pregnancy or puerperium, or if any other risk factors brought to light.

\section{References}

Jan Stam; Thrombosis of Cerebral Veins and Sinuses; N Engl J Med 2005; 352:1791-1798, April 28, 2005.

Saroja AO, Tapsi C, Naik KR; Cerebral venous thrombosis in women from Indian subcontinent; Journal of the Scientific Society;44:20-5; 2017

Murthy KK, Chefitz DL; J Clin; Cerebral Venous Sinus Thrombosis: A Case Report with Review of Diagnosis and Treatment Strategies; Case Rep 4: 354, vol 4, issue 4, 2014

Chacham S, Mukhopadhyay K, Ahluwalia J, Singh S, Kumar P, Nagasravani J; Venous Sinus Thrombosis in A Preterm Male Neonate with Elevated Anti-Phospholipid Antibodies: A Case Report; SciForchen Open Hub for Scientific Research; Open access vol 2.1; 2016.

Umesh G Rajoor et B N Seema; Cerebral Venous Thrombosis in Women: A Study from Teaching Hospital in North Karnataka; International Journal of Scientific Study; volume 3; August 2015.

Karasu D, Yilmaz C, Solak HE, Kilic I, Ali A; Cerebral Vein Thrombosis after Spinal Anaesthesia with Pregnancy; Turk J Anaesth Reanim; 43: 58-61; 2015

Zhu-Wei Liang, Gae WL, Feng LM; Clinical characteristics and prognosis of cerebral venous thrombosis in Chinese women during pregnancy and puerperium; Scientific Reports | 7:43866; www.nature.com/scientificreports; March 2017

Monteiro AMV, Amaral CM, Riberio EB, Lins MC, Miranda S, Mirenda LE ; Imaging diagnosis and clinical findings of cerebral venous thrombosis in full-term neonates without brain damage: a ten-year review; Radiol Bras;43(3):149-153; Mai/Jun 2010

Degirmenci Y, Kececi H, Seker IS;Cerebral sinus venous thrombosis in puerperium: Review of the literature in the light of two cases; Y. Cumhuriyet Medical Journal, Volume: 37, Number 1, March 2015.

Gupta RK, Jamjoom AAB, Dekota UP; Superior sagittal sinus thrombosis presenting as a continuous headache: a case report and review of the literature; Cases Journal 2:9361, December 2009 .

Chaudhuri SR, Das D, Bhattacharya S, Chakraborty S, Bhattacharjee K; Hyper Homocysteinemia Related Cerebral Venous Sinus Thrombosis -Presenting as Generalised Tonic-Clonic Seizure -A Case Report; International Journal of Clinical and Experimental Neurology, Vol. 5, No. 1, 5-8; 2017.

R. Kumar Sahoo, Tripathy P, Praharaj HN; Cerebral venous sinus thrombosis with nontraumatic subdural hematoma; International Journal of Critical Illness and Injury Science | Vol. 5 | Issue 1 | Jan-Mar 2015.

H. Allroggen et R.J Abbott; Cerebral venous sinus thrombosis; Postgrad Med J 2000;76:12-15; The Fellowship of Postgraduate Medicine, 2000.

Bansal H, Chaudhary A, Mahajan A, Paul B; Acute subdural hematoma secondary to cerebral venous sinus thrombosis: Case report and review of literature; Asian Journal of Neurosurgery;11:177; 2016

Albert Lu, Peter Y.S, Dahlin BC, Nidecker AE, Nundkumar A, Lee PS; Cerebral venous thrombosis and infarct: Review of imaging manifestations; Applied Radiology www.appliedradiology.com; 2016 
Gupta R, Aggarwal M, Patil S, Vyas V; Cerebral Venous Thrombosis Associated With Pregnancy: A Case Report; IOSR Journal of Dental and Medical Sciences (IOSR-JDMS); Volume 14, Issue 5 Ver. VII; May. 2015.

Hoffman Z, Lewis N, Chuidian FX; Cerebral Venous Sinus Thrombosis; JSM Intern Med 2(1):1003; 31st January 2017.

Lee EJ, Noh SM, Kang DW, Kim JS, Kwon SU; Impact of Provoking Risk Factors on the Prognosis of Cerebral Venous Thrombosis in Korean Patients; http://dx.doi.org/10.5853/jos.2015.01669 ; Journal of Stroke 18(2):187-194; 2016. 